Altered arterial function in migraine of recent onset was demonstrated using ultrasound and applanation tonometry in 50 patients and 50 controls, at Ghent University, Belgium. Brachial artery diameter was decreased, while carotid arterial wall properties were unchanged during a headache-free interval. Flow-mediated vasodilation of the brachial artery was decreased in patients with migraine for $>1 \mathrm{yr}$ and $<6$ yrs duration, indicative of endothelial vasomotor dysfunction. (Vanmolkot FH, Van Bortel LM, de Hoon JN. Neurology April 2007;68:1563-1570). (Tietjen GE. Editorial. Migraine as a systemic disorder. Neurology April 2007;68:1555-1556).

\title{
MANAGEMENT OF ACUTE MIGRAINE IN CHILDREN
}

The management of acute pediatric migraine headaches in the pediatric emergency department (ED) and in a mixed pediatric and adult ED was compared at the University of Alberta, Edmonton, Canada. Of 382 patients identified with headache, aged 5 to 17 years, in 4 regional EDs during the 2003/2004 fiscal year, 186 (48.7\%) met criteria for migraine. Simple oral analgesics in $20.7 \%$ and dopamine antagonists (metoclopramide and prochlorperazine) in $20.7 \%$ were prescribed first-line most commonly. Opiate medications (in 5.5\%), ketorolac $(4.7 \%$ ), and dihydroergotamine $(1 \%)$ were prescribed first-line infrequently. No treatment was given to $44.2 \%$. The pediatric ED was more likely to prescribe a dopamine antagonist $(12.9 \%$ vs $6.8 \%$; $\mathrm{P}=0.044)$ while the mixed adult/pediatric EDs were significantly more likely to prescribe an opiate $(28.1 \%$ vs $18.4 \% ; \mathrm{P}=0.031)$. Children with migraine in all EDs were significantly more likely to receive drug therapy $(68.3 \%$ vs $42.9 \% ; \mathrm{P}<0.001)$ or a dopamine antagonist $(32.3 \%$ vs $9.7 \% ; \mathrm{P}<0.001)$. Polypharmacy was used in $31.2 \%$, and neuroimaging was obtained in $29.1 \%$. In 109 studies, arachnoid cysts were diagnosed in 2 patients, optic glioma (1), sinus thrombosis (1), and sinusitis (1), a total of $5(4.6 \%)$ abnormal scans. Lumbar puncture was performed in 9 patients $(2.4 \%)$, with no difference between pediatric and adult cohorts. A headache associated infectious illness was diagnosed in $12 \%$. Documentation of headache outcome was poor, but better in pediatric EDs compared with adult EDs ( $22.1 \%$ vs $13.6 \%)$. Complete headache resolution occurred in $13.9 \%$ and was significantly more common in the pediatric ED cohort compared with the adult ED cohort ( $20 \%$ vs $4 \%$ ). (Richer L, Graham L, Klassen T, Rowe B. Emergency department management of acute migraine in children in Canada: a practice variation study. Headache May 2007;47:703-710). (Respond: Dr Lawrence Richer, University of Alberta-Pediatrics/Division of Neurology, 2C3, WMC 8440-112 St, Edmonton, Alberta, T6G 2R7, Canada).

COMMENT. Most children attending an ED in Canada with headache do not receive drug therapy. The type of medication most commonly prescribed is different in pediatric EDs compared to mixed adult/pediatric EDs. Dopamine antagonists are preferred in pediatric EDs and opiates in mixed EDs. 